\title{
Inhibition of Hes1 enhances lapatinib sensitivity in gastric cancer sphere-forming cells
}

\author{
LUCHUN LI $^{1}$, YAN LI $^{1}$, LULU WANG $^{1}$, ZHIJUAN WU ${ }^{1}$, HUIWEN MA $^{1}$, \\ JIANGHE SHAO $^{1}$, DAIRONG LI ${ }^{1}$, HUIQING YU ${ }^{1}$, WEIQI NIAN ${ }^{2}$ and DONGLIN WANG ${ }^{1}$ \\ ${ }^{1}$ Department of Oncology; ${ }^{2}$ Chongqing Key Laboratory of Translational Research for Cancer Metastasis and \\ Individualized Treatment, Chongqing Cancer Institute \& Hospital \& Cancer Center, Chongqing 400030, P.R. China
}

Received November 14, 2015; Accepted May 26, 2017

DOI: $10.3892 / 01.2017 .6683$

\begin{abstract}
It has been considered that the neurogenic locus notch homolog protein (Notch) signaling pathway serves an essential role in cellular differentiation, proliferation and apoptosis. However, the function of the Notch signaling pathway in gastric cancer stem cells (GCSCs) and epidermal growth factor receptor-tyrosine kinase inhibitor (EGFR-TKI) sensitivity remains unclear. The present study aimed to delineate the role of the Notch1 signaling pathway in GCSCs and lapatinib sensitivity. Sphere-forming cells were separated from human gastric cancer MKN45 parental cells. The sphere-forming cells exhibited characteristics of CSCs and higher Notch1 expression compared with that of parental cells. To investigate the role of the Notch1 signaling pathway in GCSCs, the expression of transcription factor Hes1 (Hes1) was knocked down using small interfering RNA against Hes1. It was observed that Hes1 expression was significantly downregulated in knocked down cells. The inhibition of Hes 1 suppressed the properties of CSCs, as indicated by significant decreases in the expression of the transcription factor sex determining region Y-box 2, epithelial cell adhesion molecule and the homeobox protein Nanog and reduced spheroid colony formation. In addition, epithelial-mesenchymal transition was significantly impaired in sphere-forming cells following Hes1 knockdown. Furthermore, the inhibition of Hes1 effectively enhanced lapatinib sensitivity in sphere-forming cells. These results suggest that sphere-forming gastric cancer cells possess the characteristics of CSCs, and that the Notch1 signaling pathway serves an essential role in the maintenance of CSCs and lapatinib sensitivity.
\end{abstract}

Correspondence to: Professor Donglin Wang, Department of Oncology, Chongqing Cancer Institute \& Hospital \& Cancer Center, 181 Hanyu Road, Chongqing 400030, P.R. China

E-mail: 676881521@qq.com

Key words: neurogenic locus notch homolog protein 1, gastric cancer, cancer stem cells, epithelial-mesenchymal transition, epidermal growth factor receptor-tyrosine kinase inhibitor

\section{Introduction}

Epidermal growth factor receptor (EGFR) and human EGFR2 (HER2) are frequently upregulated or mutated in various types of cancer, and serve essential roles in cancer cell proliferation, survival, migration and differentiation $(1,2)$. EGFR is frequently mutated or overexpressed in lung, brain, colon, pancreatic and breast cancer (3-6). Furthermore, HER2 is often overexpressed in breast, gastric, esophageal, pancreatic and ovarian cancer $(7,8)$. EGFR-tyrosine kinase inhibitors (TKIs), including gefitinib and erlotinib, are used either alone or in combination with radiation or chemotherapy in cancer therapy (9). The EGFR-TKI lapatinib, and the monoclonal antibody trastuzumab have been approved for the treatment of HER2-overexpressing breast cancer (10). Lapatinib is a potent adenosine trisphosphate (ATP)-competitive dual kinase inhibitor that inhibits EGFR and HER2, and has demonstrated antiproliferative activity against human HER2-amplified breast cancer cell lines (11). Wainberg et al (12) reported that lapatinib selectively inhibits the proliferation of HER2-amplified human gastric cancer cells. However, lapatinib is currently unsatisfactory for the treatment of patients with gastric cancer (13). A previous clinical trial suggested that HER2-targeted therapy is associated with drug resistance in cancer cells (14). However, it is unknown whether such resistance mechanisms are associated with gastric cancer stem cells (GCSCs).

The CSC hypothesis was introduced to explain the pathogenesis of numerous cancer types. CSCs have the ability to self-renew and proliferate indefinitely, which can initiate tumor formation and cause tumor recurrence. CSCs are distinguished from the bulk of the tumor cell population by their ability to successfully seed new tumors when implanted in low numbers into experimental animals (15). Furthermore, CSCs are more resistant to chemotherapy and radiotherapy than their corresponding differentiated cancer cells (16-18). The neurogenic locus notch homolog protein (Notch) signaling pathway serves an important role in the determination of cell fate in various organ systems (19). The Notch pathway encompasses four types of receptors (Notch1, $-2,-3$ and -4 ) and five membrane proteins ligands that include Delta-like ligands (DLL1, -2 and -3) and Serrate/Jagged (JAG1 and -2). The Notch1 signaling pathway is essential in maintaining the characteristics of CSCs and is associated 
with the self-renewal of various types of CSCs, including breast and pancreatic CSCs (20). Hes1 is the downstream target gene of Notch1 pathway (21). Hes1 may have an important function in the maintenance of cancer stem cells self-renewal and epithelial-mesenchymal transition (EMT) process (22).

In the present study, the role of the Notch1 signaling pathway in GCSCs and lapatinib sensitivity was examined. Furthermore, the current study aimed to elucidate the molecular mechanism underlying resistance to lapatinib in GCSCs.

\section{Materials and methods}

Culture of sphere-forming cells. MKN45 cells were purchased from the Type Culture Collection of the Chinese Academy of Sciences (Shanghai, China), which were seeded at a density of $2 \times 10^{4}$ cells $/ \mathrm{ml}$ in $100 \mathrm{~mm}$ ultralow attachment plates (Costar; Corning Incorporated, Corning, NY, USA). Cells were grown in serum-free RPMI-1640 and Nutrient Mixture F-12 medium (both Hyclone; GE Healthcare Life Sciences, Logan, UT, USA) supplemented with B27 (1:50; Hyclone; GE Healthcare Life Sciences), $20 \mathrm{ng} / \mathrm{ml} \mathrm{EGF,} 20 \mathrm{ng} / \mathrm{ml}$ basic fibroblast growth factor (bFGF) (both R\&D Systems, Minneapolis, MN, USA), $5 \mu \mathrm{g} / \mathrm{ml}$ bovine insulin (Cell Applications, Inc., San Diego, CA, USA), $0.5 \mu \mathrm{g} / \mathrm{ml}$ hydrocortisone (Sigma-Aldrich; Merck KGaA, Darmstadt, Germany) and penicillin/streptomycin (Hyclone; GE Healthcare Life Sciences). MKN45 cells were grown for 3 days in the above sphere culture maintained at $37^{\circ} \mathrm{C}$ in a $5 \% \mathrm{CO}_{2}$ atmosphere and produced spheres, which were dissociated through incubation with $0.05 \%$ trypsin/EDTA (Sigma-Aldrich; Merck KGaA) on day 4. Dissociated MKN45 cells were cultured in the aforementioned sphere conditions maintained at $37^{\circ} \mathrm{C}$ in a $5 \% \mathrm{CO}_{2}$ atmosphere for another 3 days and then harvested.

Tumorigenicity assay. A total of 404 -week-old female nude mice (weight 18-20 g) were obtained from the Shanghai Experimental Animal Center of the Chinese Academy of Science (Shanghai, China). The mice, were given SPF grade feed and purified water and were kept in cages (5 mice/cage) in a room with a constant temperature $\left(22 \pm 1^{\circ} \mathrm{C}\right)$ and a $12 \mathrm{~h}$ dark/light cycle. For in vivo experiments, sphere-forming and parental cells were resuspended in PBS (Hyclone; GE Healthcare Life Sciences), and injected subcutaneously into the limbs of mice. The protocol used in the present study was approved by the Ethics Committee of Chongqing Cancer Institute (Chongqing, China). Groups of mice were inoculated with sphere-forming or parental cells at $5 \times 10^{2}, 5 \times 10^{3}, 5 \times 10^{4}$ or $5 \times 10^{5}$ (five mice/group). Tumor growth was monitored every 2 days following the second week of inoculation.

Lentivirus transfection. Inhibition of transcription factor Hes1 (Hes1) was achieved by infecting cells with the Hes1-small interfering (si)RNA lentivirus (Shanghai GenePharma Co., Ltd., Shanghai, China). Transduction was performed using a GenePharma Lentivirus Transduction kit (Shanghai GenePharma Co., Ltd.). The target sequence for the Hes1 siRNA was 5'-AGATCAATGCCATGACCTA-3'. Cells were cultured in six-well plates at 20-30\% confluence and incubated for $12 \mathrm{~h}$ at $37^{\circ} \mathrm{C}$ with $5 \% \mathrm{CO}_{2}$. Cells were then infected with Hes1-siRNA- or scrambled control-siRNA-expressing lentiviruses $\left(5 \times 10^{8} \mathrm{TU} / \mathrm{ml}, 40 \mu \mathrm{l}\right.$, Shanghai GenePharma Co.,Ltd.). Following the infections, the culture medium was replaced with supernatant fluid that contained an appropriate viral titer $(1 \mathrm{ml} /$ well $)$. After incubating at $37^{\circ} \mathrm{C}$ for $12 \mathrm{~h}$, the viral supernatant was replaced with fresh medium. The infected cells were selected using $2 \mathrm{mg} / \mathrm{ml}$ puromycin (Santa Cruz Biotechnology, Inc., Dallas, TX, USA) following incubation for $48 \mathrm{~h}$. Successful infection was confirmed by expression of green fluorescent protein using an inverted fluorescence microscope (Leica DMI4000 B; Leica Microsystems GmbH, Wetzlar, Germany). The knockdown efficiency was determined using western blotting as described below.

Spheroid colony formation assay. Cells were inoculated into each well (20 cells/well) of ultralow attachment 48-well plates (Costar; Corning Incorporated) and supplemented with $300 \mu 1$ of RPMI-1640 containing $40 \mathrm{ng} / \mathrm{ml} \mathrm{bFGF}$ and $20 \mathrm{ng} / \mathrm{ml} \mathrm{EGF}$. After incubation for 4 weeks at $37^{\circ} \mathrm{C}$ with $5 \% \mathrm{CO}_{2}$, the total number of spheroid colonies/well was counted.

Cell chemosensitivity examination. Cells cultured in medium were incubated at $37^{\circ} \mathrm{C}$ with $5 \% \mathrm{CO}_{2}$ and treated with $6 \mathrm{mM}$ 5-fluorouracil (5-Fu) or $5 \mu \mathrm{mol} / 1$ lapatinib (both Sigma-Aldrich; Merck KGaA). After $48 \mathrm{~h}$ of exposure, $20 \mathrm{ml}$ of $0.5 \mathrm{mg} / \mathrm{ml} \mathrm{MTT}$ solution (Sigma-Aldrich; Merck KGaA) was added for an additional $4 \mathrm{~h}$ and then $100 \mathrm{ml}$ dimethyl sulfoxide (Sigma-Aldrich; Merck KGaA) was added for $15 \mathrm{~min}$. The plates were agitated at a low speed for $5 \mathrm{~min}$ and the absorbance was measured at a wavelength of $570 \mathrm{~nm}$ using a spectrophotometer. Five wells were assayed for each condition.

Western blotting. According to the manufacturer's protocol, total protein samples for immunoblots were extracted from cells using radioimmunoprecipitation assay lysis buffer (Beyotime Institute of Biotechnology, Shanghai, China). Following quantification of the protein extracts using a BCA protein assay, equivalent amounts of protein (40 $\mu \mathrm{g} / \mathrm{lane})$ were resolved using 10\% SDS-PAGE and then transferred onto a polyvinylidene fluoride membrane (both Beyotime Institute of Biotechnology). Membranes were subsequently blocked with 5\% non-fat milk in TBS-Tween-20 (Beyotime Institute of Biotechnology) for $1 \mathrm{~h}$ at $4^{\circ} \mathrm{C}$. Next, the blots were incubated with the appropriate primary antibody for $12 \mathrm{~h}$ at $4^{\circ} \mathrm{C}$ and then with the corresponding horseradish peroxidase (HRP)-conjugated secondary antibody for $2 \mathrm{~h}$ at $37^{\circ} \mathrm{C}$. Signals were detected using an enhanced chemiluminescence reagent (EMD Millipore, Billerica, MA, USA). The results were analyzed by Quantity One software (version 4.6.2, Bio-Rad Laboratories, Inc., Hercules, CA, USA).

Antibodies directed against GAPDH (cat. no. 560005) and zinc finger protein SNAI1 (Snail, cat. no. 550589) were purchased from BD Biosciences (Franklin Lakes, NJ, USA). Antibodies directed against epithelial cell adhesion molecule (EpCAM, cat. no. ab71916), the homeobox protein Nanog (Nanog, cat. no. ab109250), the transcription factor sex determining region Y-box 2 (Sox2, cat. no. ab92494), multidrug resistance protein 1 (MDR1, catalog no. ab170904), ATP-binding cassette sub-family G member ABCG1 
(cat. no. ab52617), ABCG2 (cat. no. ab24115), DNA repair protein RAD51 homolog 1 (RAD51, cat. no. ab88572), tight junction protein ZO-1 (ZO1, cat. no. ab59720), N-cadherin (cat. no. ab76057), E-cadherin (cat. no. ab1416) and vimentin (cat. no. ab92547) were purchased from Abcam (Cambridge, UK). The monoclonal goat anti-rabbit IgG and goat anti-mouse HRP-conjugated antibodies (cat. no. sc-2354) were purchased from Santa Cruz Biotechnology Inc.

The following antibody dilutions were used: Anti-Notch1, 1:1,200; anti-Hes1, 1:10,000; anti-E-cadherin, 1:1,200; anti-N-cadherin, 1:1,200; anti-vimentin, 1:1,200; anti-Snail, 1:500; anti-ZO1, 1:500; anti-EpCAM, 1:1,500; anti-Nanog, 1:1,500; anti-Sox 2, 1:1,500; anti-MDR1, 1:1,000; anti-ABCG1, 1:1,000; anti-ABCG2, 1:1,000; anti-RAD51, 1:500; anti-GAPDH, 1:500; and HRP-conjugated IgG antibodies, $1: 7,000$.

Reverse transcription-quantitative polymerase chain reaction (RT-qPCR). RNA was extracted from the cells using RNAiso (Takara Bio, Inc., Otsu, Japan) and complementary DNA (cDNA) was then synthesized using the PrimeScript II First Strand cDNA Synthesis kit (Takara Bio, Inc.) according to the manufacturers' protocols. PCR (100 ng cDNA used per qPCR) was performed using a CFX96 ${ }^{\text {TM }}$ Real-Time PCR Detection System (Bio-Rad Laboratories, Inc.) with SYBR ${ }^{\circledR}$ Premix Ex Taq ${ }^{\mathrm{TM}}$ II (Takara Bio, Inc.). The PCR conditions were as follows: $95^{\circ} \mathrm{C}$ for $30 \mathrm{sec}$, followed by 40 cycles of $95^{\circ} \mathrm{C}$ for $5 \mathrm{sec}$ and $60^{\circ} \mathrm{C}$ for $30 \mathrm{sec}$. Data were normalized against $\beta$-actin messenger RNA (mRNA). The sequences of the PCR primers were as follows: Notch1 forward, 5'-TGC CGAACCAATACAACCCTC-3' and reverse, 5'-TGGTAG CTCATCATCTGGGACA-3'; Hes1 forward, 5'-GTGCAT GAACGAGGTGACCC-3' and reverse, 5'-GTATTAACG CCCTCGCACGT-3'; and $\beta$-actin forward, 5'-CCACGA AAC TACCTTCAACTCC-3' and reverse, 5'-GTGATCTCCTTC TGCATCCTGT-3'. qPCR was performed according to the $2^{-\Delta \Delta \mathrm{Cq}}$ method (23).

Histological examination. Tumor tissues were fixed in $10 \%$ neutral-buffered formalin for $24 \mathrm{~h}$ at room temperature, embedded in paraffin, and then the sections (5 um) were stained with hematoxylin for $10 \mathrm{~min}$ and eosin (both Beyotime Institute of Biotechnology) for $5 \mathrm{sec}$ at room temperature. Histological differences were examined using an inverted microscope.

Statistical analysis. All experiments were repeated three times and the results were analyzed using SPSS 16.0 software (SPSS, Inc., Chicago, IL, USA). Data are presented as the mean \pm standard deviation. The statistical significance of the differences among the groups was evaluated using the Student's t-test (two-tailed). $\mathrm{P}<0.05$ was considered to indicate a statistically significant difference.

\section{Results}

Sphere-forming cells isolated from gastric cancer MKN45 cells exhibit the characteristics of CSCs. Tumors contain a small number of CSCs that possess self-renewal and tumor-initiating abilities (24). To isolate sphere-forming cells,
Table I. Tumorigenicity of sphere-forming and parental cells in nude mice ${ }^{\mathrm{a}}$.

\begin{tabular}{lcccc}
\hline & \multicolumn{4}{c}{ No. of cells injected } \\
\cline { 2 - 5 } Tumor marker presence & $5 \times 10^{2}$ & $5 \times 10^{3}$ & $5 \times 10^{4}$ & $5 \times 10^{5}$ \\
\hline CD44 $^{+}$ & $2 / 5$ & $4 / 5$ & $5 / 5$ & $5 / 5$ \\
CD44- $^{-}$ & $0 / 5$ & $0 / 5$ & $0 / 5$ & $1 / 5$ \\
\hline
\end{tabular}

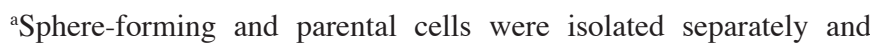
injected subcutaneously into nude mice. Tumor formation was observed for 8 weeks following injection. Each experiment was repeated five times. The results represent the number of mice that developed a tumor. $\mathrm{CD}$, cluster of differentiation.

MKN45 cells were grown in ultralow attachment plates with sphere culture medium for 7 days. Subsequently, the expression levels of CSC markers, including EpCAM, Nanog and Sox 2, were determined using western blotting. Concordant with previous studies the protein expression levels of these CSC markers were significantly higher in sphere-forming cells than in parental cells (Fig. 1A). Furthermore, in the spheroid colony formation assay, sphere-forming cells formed significantly more spheroids compared with those in parental cells (Fig. 1B).

In the tumorigenicity assay, nude mice were injected with $5 \times 10^{2}-5 \times 10^{5}$ sphere-forming or parental cells. Transplantation of $5 \times 10^{2}, 5 \times 10^{3}$ or $5 \times 10^{4}$ parental cells consistently failed to form tumors in all mice, while $5 \times 10^{5}$ parental cells led to tumor formation in 1/5 mice (Table I). By contrast, transplantation of $5 \times 10^{2}$ sphere-forming cells resulted in tumor formation in $2 / 5$ mice, while transplantation of $5 \times 10^{3}, 5 \times 10^{4}$ or $5 \times 10^{5}$ sphere-forming cells resulted in tumor formation in all mice (Table I). Subsequently, the chemotherapy susceptibility of sphere-forming and parental cells to 5-Fu, which is generally used for the treatment of gastric cancer, was examined. Sphere-forming cells were significantly more chemoresistant to 5-Fu than parental cells, as determined using an MTT cell viability assay (Fig. $1 \mathrm{C} ; \mathrm{P}<0.05$ ). These data suggest that sphere-forming cells are tumorigenic and possess CSC characteristics.

Notchl signaling pathway is activated in sphere-forming MKN45 cells. To investigate the role of the Notch1 signaling pathway in CSCs, the expression of Notch1 and Hes1 in sphere-forming and parental cells was analyzed. It was revealed that Notch1 and Hes1 mRNA and protein expression levels were significantly higher in sphere-forming cells compared with those in parental cells (Fig. 2). These data suggest that the Notch1 signaling pathway is activated in sphere-forming cells.

Sphere-forming MKN45 cells are resistant to the EGFR-TKI lapatinib. The sensitivities of sphere-forming and parental cells to lapatinib were next assessed. The results of the MTT assays demonstrated that the viability of sphere-forming cells was significantly increased compared with that of parental cells $(\mathrm{P}<0.05$; Fig. 3$)$. Therefore, this suggests that 
A
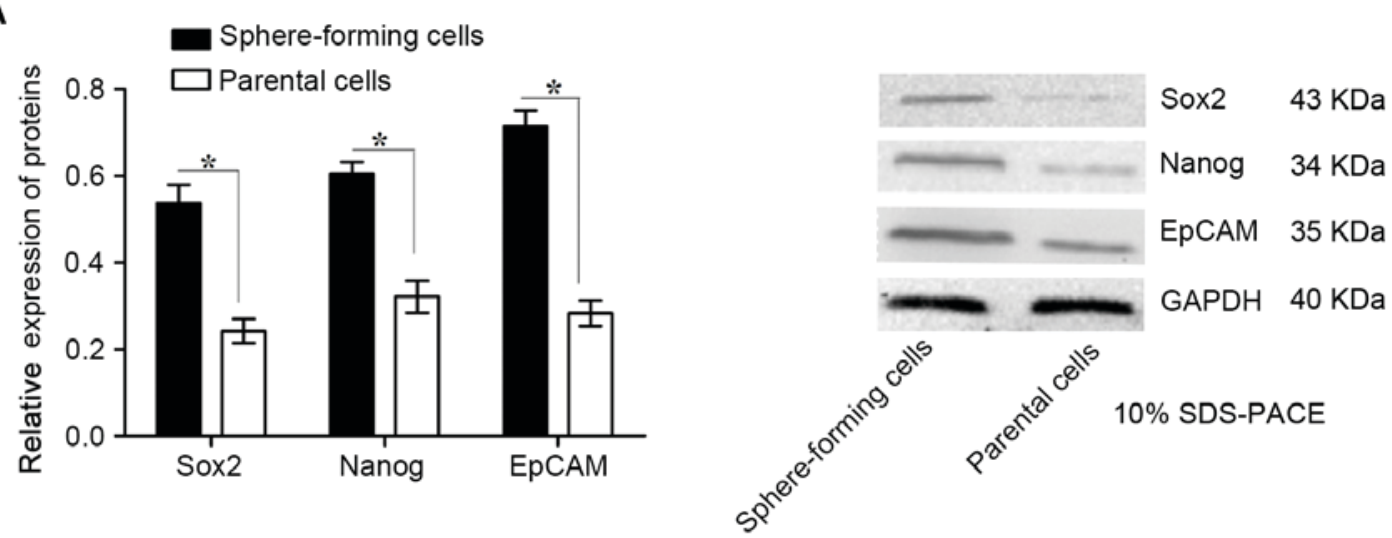

B

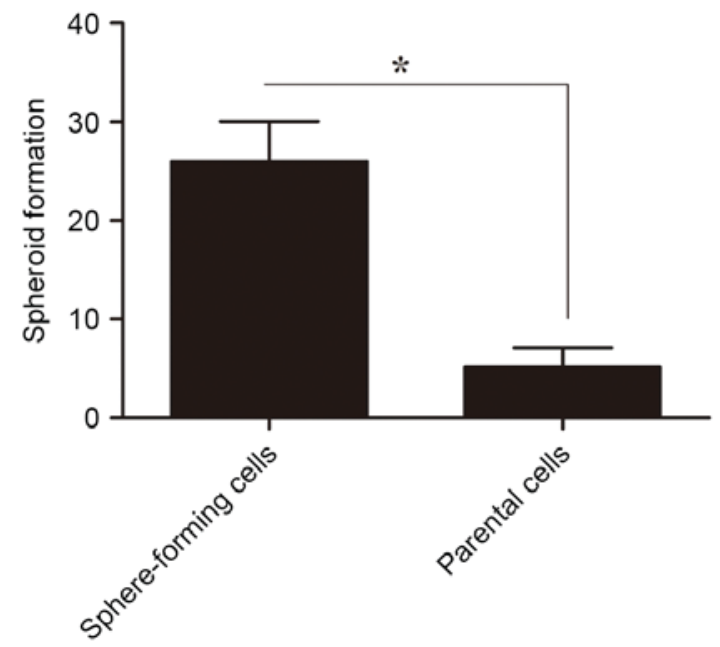

C

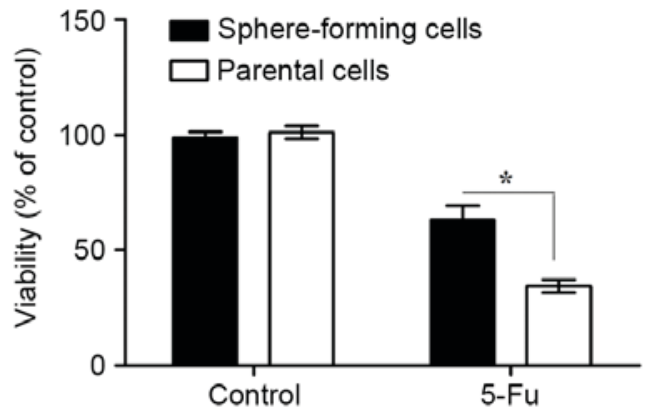

Figure 1. Expression of CSC markers, spheroid-forming ability and chemotherapy susceptibility of sphere-forming and parental cells isolated from MKN45 cells. (A) CSC markers, including EpCAM, Nanog and Sox2, were evaluated using western blotting relative to GAPDH. The protein expression levels of these CSC markers were significantly higher in sphere-forming cells compared with those in parental cells. (B) Sphere-forming cells formed significantly more spheroids than parental cells. (C) Sphere-forming cells were significantly more chemoresistant to 5-Fu than parental cells, as indicated by their cell viability levels determined using an MTT assay. Data are presented as the mean \pm standard deviation ( $\mathrm{n}=5$ ). " $\mathrm{P}<0.05$. CSC, cancer stem cell; EpCAM, epithelial cell adhesion molecule; Nanog, homeobox protein Nanog; Sox2, sex determining region Y-box 2; 5-Fu, 5-fluorouracil.
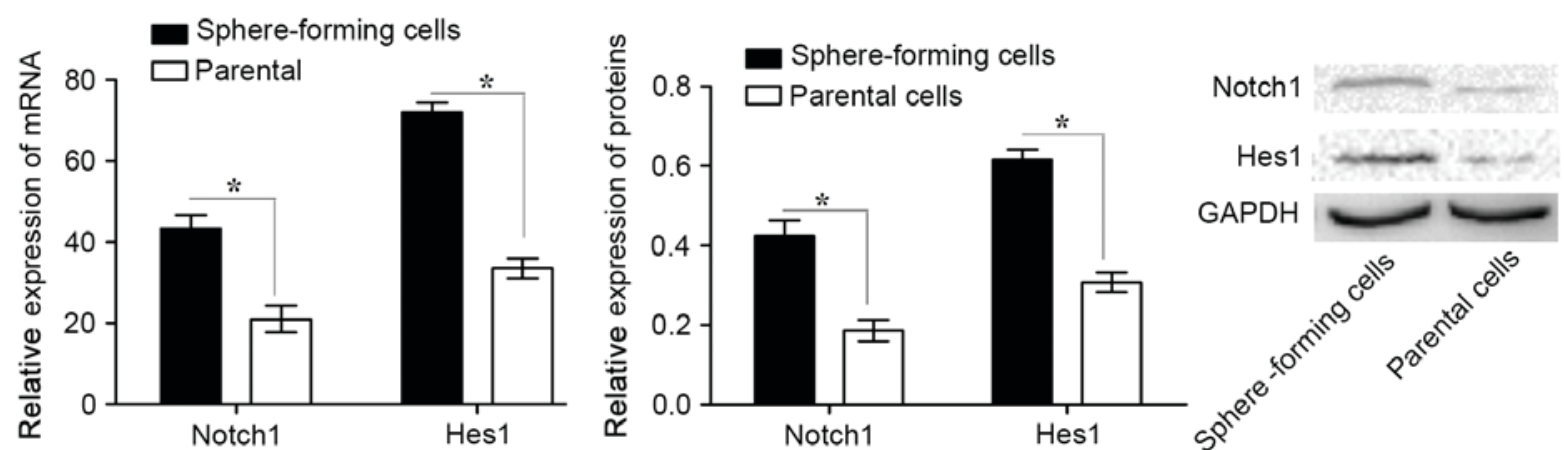

Figure 2. The Notch1 signaling pathway is activated in sphere-forming cells. The mRNA and protein expression levels of Notch1 and Hes1 were significantly higher in sphere-forming cells compared with those in parental cells. mRNA and protein expression levels were normalized against $\beta$-actin and GAPDH, respectively. Data are presented as the mean \pm standard deviation $(n=5)$. "P<0.05. Notch1, neurogenic locus notch homolog protein 1; Hes1, transcription factor Hes1; mRNA, messenger RNA.

sphere-forming cells are more resistant to lapatinib than parental cells.

Inhibition of Hesl expression attenuates the characteristics of CSCs and resistance to lapatinib in sphere-forming cells.
To examine the effects of the Notch1 signaling pathway in GCSCs, sphere-forming cells were infected with lentivirus containing Hes1-siRNA or scrambled control-siRNA. The protein expression levels of Hes1 were determined using western blot analysis. Infection of sphere-forming cells with 


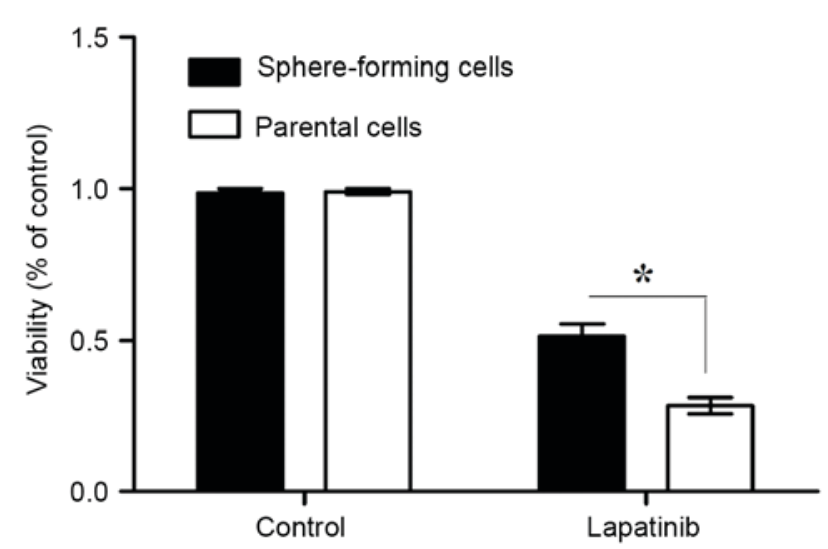

Figure 3. Sphere-forming cells were significantly more resistant to lapatinib than parental cells, as determined using an MTT cell viability assay. Data are presented as the mean \pm standard deviation $(n=5)$. ${ }^{*}<<0.05$.

lentivirus containing Hes1-siRNA significantly reduced Hes1 protein expression levels compared with those in control-siRNA-treated and untreated cells, while the control siRNA group exhibited no significant effect compared with the untreated control group (Fig. 4A). Simultaneously, the protein expression levels of the CSC markers EpCAM, Nanog and Sox 2 were significantly downregulated in Hes1-siRNA lentivirus-infected sphere-forming cells compared with those in the control-siRNA group (Fig. 4B).

In the spheroid colony formation assay, sphere-forming cells infected with Hes1-siRNA lentivirus formed significantly less spheroids compared with those in the control-siRNA lentivirus-infected cells (Fig. 4C). In addition, the results of the MTT assay revealed that the susceptibility of Hes1-siRNA lentivirus-infected cells to the chemotherapeutic agent $5-\mathrm{Fu}$ was significantly increased compared with that of the control-siRNA group (Fig. 4D).

To investigate the influence of the Notch1 signaling pathway on the susceptibility of sphere-forming cells to EGFR-TKIs, the susceptibility of Hes1-siRNA lentivirus-infected sphere-forming cells to lapatinib was assessed. Data from the MTT assays demonstrated that the survival rate of Hes1-siRNA lentivirus-infected sphere-forming cells was significantly decreased compared with that of control-siRNA lentivirus-infected cells $(\mathrm{P}<0.05$; Fig. 4E). These data indicate that inhibition of Hes1 expression attenuates the characteristics of CSCs and increases susceptibility to lapatinib in sphere-forming cells.

Inhibition of Hesl prevents EMT and decreases the expression of chemoresistance-associated proteins in sphere-forming MKN45 cells. To further investigate the molecular mechanisms of the Notch1 signaling pathway on the susceptibility of sphere-forming cells, the expression of EMT markers and chemoresistance-associated proteins was examined. Western blot analysis demonstrated that the expression levels of the epithelial markers E-cadherin and ZO1 were significantly upregulated, while the expression levels of the mesenchymal markers $\mathrm{N}$-cadherin, vimentin and Snail were significantly downregulated in Hes1-siRNA lentivirus-infected sphere-forming cells compared with those in the control-siRNA group (Fig. 5A). Furthermore, the expression levels of the chemoresistance-associated proteins MDR1, ABCG1, ABCG2 and RAD51 were significantly decreased in Hes1-siRNA lentivirus-infected sphere-forming cells (Fig. 5B). Together, these results indicate that inhibition of Hes1 can impair EMT and decrease the expression of chemoresistance-associated proteins in sphere-forming MKN45 cells.

\section{Discussion}

The CSC hypothesis suggests that cancer is maintained by a subpopulation of stem cells with an indefinite life span, which raises the possibility that targeting CSCs could provide an approach for cancer treatment (25). The sphere-forming assay in which cells are cultured in non-adherent conditions in a serum-free medium supplemented with bFGF and EGF is a practical approach for identifying stem cells in individual solid tumor tissue samples or cancer cell cultures $(26,27)$. In the present study, sphere-forming cells were developed by cultivating the human gastric cancer cell line MKN45 in defined serum-free medium. The sphere-forming cells were able to generate significantly more spheroid bodies than their parental cells. This phenomenon indicates that sphere-forming cells are capable of self-renewal and proliferation, which are important characteristics of CSCs (25). Chemoresistance is another important characteristic of CSCs (18). To assess whether the self-renewing sphere-forming cells possessed a hypothetical CSC chemoresistant property, the sensitivity of sphere-forming cells to chemotherapeutic agents was examined. Sphere-forming cells exhibited significantly greater resistance to 5-Fu compared with that of their parental cells, as determined using cell viability assays. Xenotransplantation is generally regarded as the gold standard for evaluating the tumorigenicity of tumor cells. In the tumorigenicity assay, nude mice were injected with sphere-forming or parental cells. As few as 500 sphere-forming cells were able to generate tumors in mice. Additionally, sphere-forming cells generated subcutaneous tumors with larger volumes in shorter time periods compared with those generated from parental cells. To further investigate the CSC properties of sphere-forming cells, the expression of the CSC markers Sox2, Nanog and EpCAM was investigated. Western blot analysis demonstrated that the expression levels of Sox2, Nanog and EpCAM were significantly higher in sphere-forming cells than in parental cells. In summary, sphere-forming cells from the human gastric cancer cell line MKN45 possess GCSC properties, which is in agreement with previous studies (28).

The Notch signaling pathway serves an important role in cellular processes during embryonic and postnatal development, including stem cell renewal, cell fate determination and apoptosis (15). However, dysregulation of the Notch signaling pathway also contributes to tumorigenesis (29). The interaction of Notch ligands with their receptors promotes $\gamma$-secretase-dependent cleavage of the Notch receptor and releases the Notch intracellular domain, which results in the activation of the signaling pathway and induces target genes, including Hes1 (30). The role of the Notch1 signaling pathway in the maintenance of CSCs has been described in preclinical models and in clinical studies $(31,32)$. Using gain- and 

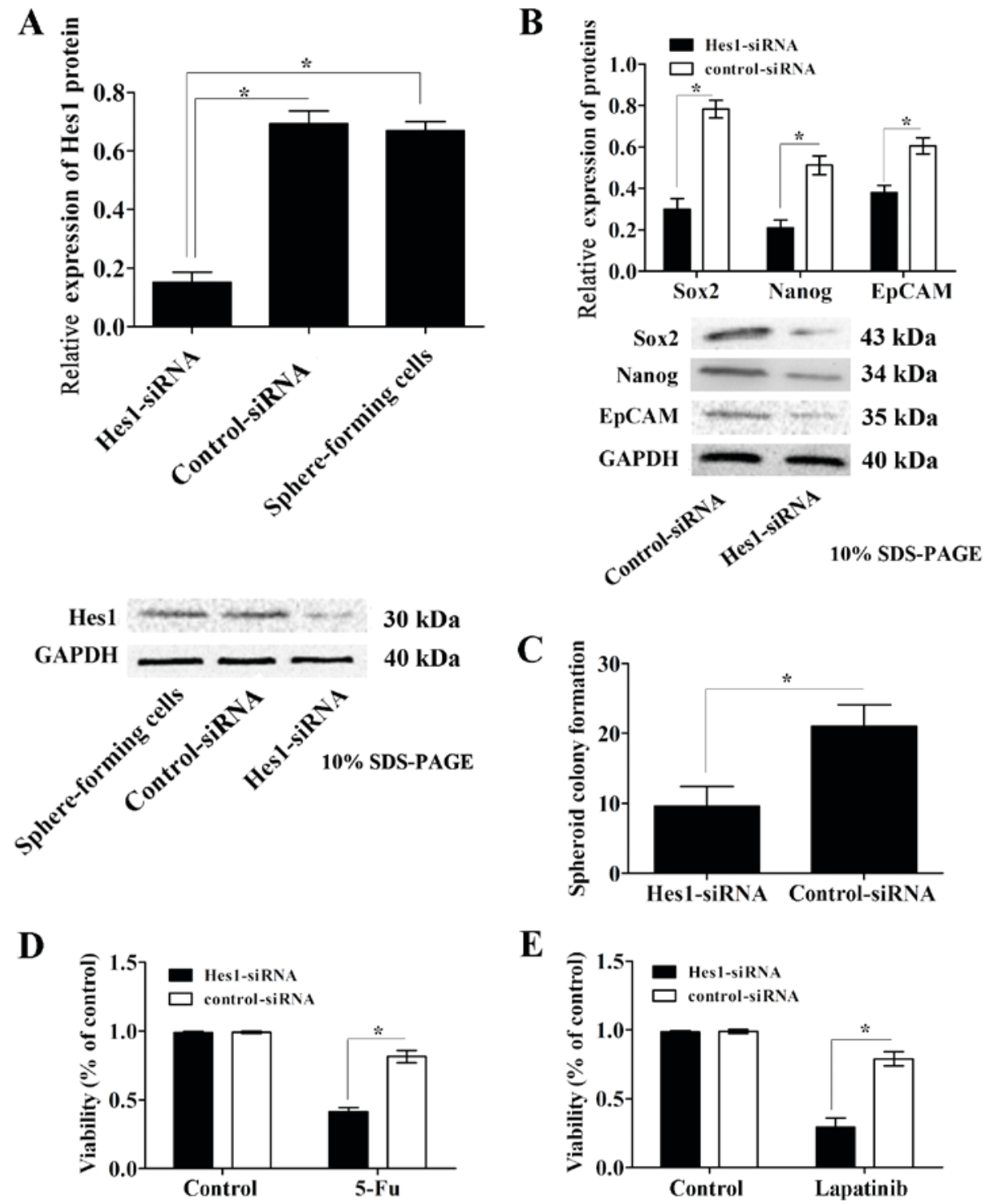

Figure 4. Inhibition of Hes1 expression attenuates the characteristics of CSCs and resistance to lapatinib in sphere-forming cells. (A) Hes1 protein expression levels were significantly downregulated in sphere-forming cells infected with lentivirus containing Hesl-siRNA compared with those in the control-siRNA infected and untreated groups. (B) The protein expression levels of the CSC markers EpCAM, Nanog and Sox2, were significantly decreased in Hes1-siRNA lentivirus-infected sphere-forming cells compared with those in the control-siRNA group. (C) Sphere-forming cells infected with Hes1-siRNA lentivirus formed significantly fewer spheroids than control-siRNA lentivirus-infected cells. The chemotherapy susceptibly of Hes1-siRNA lentivirus-infected sphere-forming cells to (D) 5-Fu and (E) lapatinib was significantly increased compared with that of control-siRNA lentivirus-infected cells. Protein expression levels were normalized to GAPDH. Data are presented as the mean \pm standard deviation $(n=5)$. ${ }^{*} \mathrm{P}<0.05$. Notch1, neurogenic locus notch homolog protein 1 ; Hes1, transcription factor Hes1; EpCAM, epithelial cell adhesion molecule; Nanog, homeobox protein Nanog; Sox2, sex determining region Y-box 2; siRNA, small interfering RNA; CSC, cancer stem cell; 5-Fu, 5-fluorouracil.

loss-of-function approaches, the maintenance of CSCs has been attributed to Notch signaling regulation through Hes1, which dictates cell fate decisions (33). In the present study, the expression of Notch1 and its downstream target Hes1 were significantly higher in sphere-forming cells compared with those in parental cells.

To the best of our knowledge, the role of the Notch1 signaling pathway in the lapatinib resistance of GCSCs has not been investigated previously. Therefore, in the present study, the sensitivities of sphere-forming and parental cells to lapatinib were assessed, and it was demonstrated that sphere-forming cells were significantly more resistant to lapatinib than parental cells. To investigate the potential molecular mechanisms that influence the Notch1 signaling pathway in lapatinib resistance, the expression of Hes1 was inhibited via transfection with Hes1-siRNA lentivirus. Following the downregulation of Hes1 expression, the CSC properties of sphere-forming cells were significantly impaired and the expression levels of CSC markers were significantly downregulated compared with those of the corresponding control siRNA groups.

The activation of EGFR family proteins is regulated by ligand binding, with the exception of HER2, which dimerizes independently of ligand binding $(34,35)$. Once dimerization occurs, intracellular tyrosine kinases are fully activated and induce autophosphorylation of their tyrosine residues. These phosphorylated tyrosines function as docking sites for several adapter proteins, including growth factor receptor bound 
A

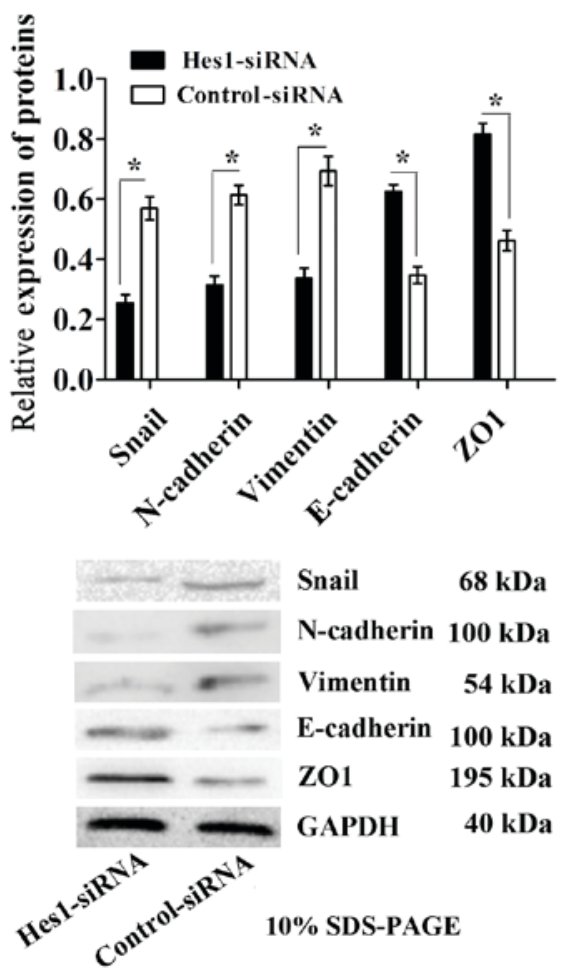

B

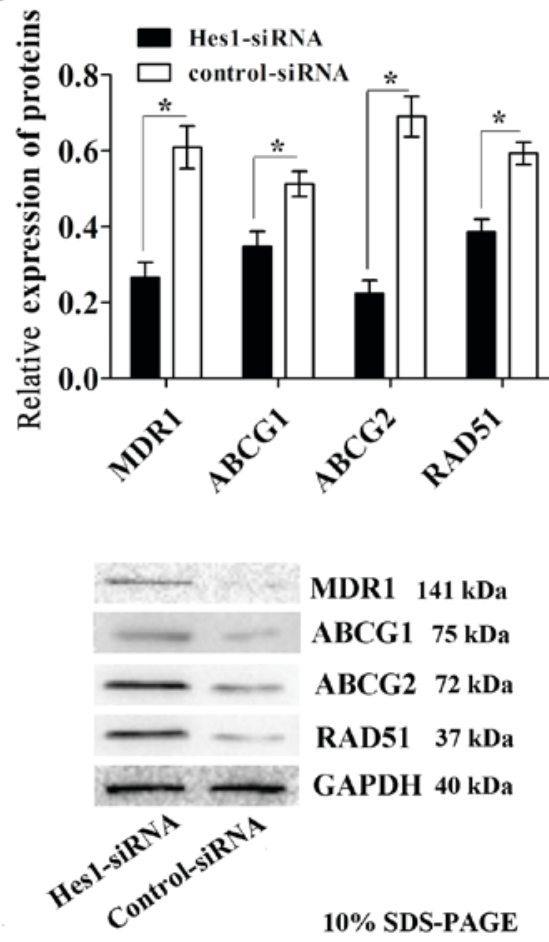

Figure 5. Infection with Hes1-siRNA lentivirus results in impaired EMT and decreased expression of chemoresistance-associated proteins in sphere-forming MKN45 cells. (A) EMT was impaired in sphere-forming cells infected with Hes1-siRNA lentivirus, as demonstrated by a significant decrease in the expression levels of the EMT-associated proteins Snail, N-cadherin and vimentin, and an increase in expression level of E-cadherin and ZO1 compared with those in the control-siRNA group. (B) The expression levels of the chemoresistance-associated proteins MDR1, ABCG1, ABCG2 and RAD51 were significantly downregulated in sphere-forming cells infected with Hes1-siRNA lentivirus compared with those in the control-siRNA group. Protein expression levels were normalized to GAPDH. Data are presented as the mean \pm standard deviation ( $\mathrm{n}=5$ ). ${ }^{*} \mathrm{P}<0.05$. Notch1, neurogenic locus notch homolog protein 1; Hes1, transcription factor Hes1; siRNA, small interfering RNA; Snail, zinc finger protein SNAI1; MDR1, multidrug resistance protein 1; ABCG, ATP-binding cassette sub-family G member; RAD51, DNA repair protein RAD51 homolog 1; ZO1, tight junction protein ZO-1; EMT, epithelial-mesenchymal transition.

protein 2 and SHC adaptor protein, which further transduce the signaling pathways through protein-protein interactions and post-translational modifications (36). Cross-regulation between EGFR/HER2 and Notch signaling pathways has long been observed in genetic studies (37). For instance, activation of the Notch1 signaling pathway is associated with EGFR-TKI resistance in PC9 cells expressing mutated EGFR (38). In the present study, MKN45 cells were selected, as HER2 is highly activated in these cells (39). It was revealed that the inhibition of Hes1 significantly increased the sensitivity of sphere-forming cells to lapatinib.

To further investigate the role of the Notch1 signaling pathway in lapatinib resistance, EMT and resistance-associated protein expression levels were examined. During EMT, epithelial cells lose several of their epithelial characteristics, including E-cadherin and ZO1 expression, and acquire properties that are typical of mesenchymal cells, including the expression of vimentin (40). In the present study, the inhibition of Hes1 decreased Snail expression and impaired EMT in sphere-forming cells. Additionally, it is known that CSCs can effectively increase drug resistance through upregulating the expression of drug efflux transporter genes and various multidrug resistance genes (41). CSCs isolated from the sphere-forming culture of cancer cells were previously demonstrated to possess high expression of the ABCB1 gene and were identified to be significantly resistant to various chemotherapeutic agents (42). In the present study, the expression levels of the resistance-associated proteins MDR1, ABCG1, ABCG2 and RAD51 were significantly downregulated following inhibition of Hes1. These results suggest that inhibiting the Notch1 signaling pathway through inhibition of Hes1 prevents evasion of CSCs from HER2-targeted therapy and potentially increases the sensitivity of GCSCs to EGFR-TKIs.

The results of the present study support the idea that sphere-forming cells isolated from human gastric cancer cells possess the properties of CSCs. Furthermore, the role of the Notch1 signaling pathway in GCSCs was demonstrated. In addition, the inhibition of Hes1 through siRNA knockdown was revealed to significantly impair the stemness of GCSCs and increase the sensitivity of GCSCs to EGFR-TKIs. In the future, a combination strategy using EGFR/HER2 and Notch signaling pathway inhibitors may become a treatment option for patients with gastric cancer.

\section{Acknowledgements}

The present study was supported by the National Natural Science Foundation of China (grant no. 81172387) and the National Natural Science Foundation of Chongqing (grant no. C2011BB5119). 


\section{References}

1. Dhomen NS, Mariadason J, Tebbutt N and Scott AM: Therapeutic targeting of the epidermal growth factor receptor in human cancer. Crit Rev Oncog 17: 31-50, 2012.

2. Baselga J and Arteaga CL: Critical update and emerging trends in epidermal growth factor receptor targeting in cancer. J Clin Oncol 23: 2445-2459, 2005.

3. Rimawi MF, Shetty PB, Weiss HL, Schiff R, Osborne CK, Chamness GC and Elledge RM: Epidermal growth factor receptor expression in breast cancer association with biologic phenotype and clinical outcomes. Cancer 116: 1234-1242, 2010.

4. Shigematsu H and Gazdar AF: Somatic mutations of epidermal growth factor receptor signaling pathway in lung cancers. Int J Cancer 118: 257-262, 2006.

5. Gan HK, Kaye AH and Luwor RB: The EGFRvIII variant in glioblastoma multiforme. J Clin Neurosci 16: 748-754, 2009.

6. Bronte G, Terrasi M, Rizzo S, Sivestris N, Ficorella C, Cajozzo M, Di Gaudio F, Gulotta G, Siragusa S, Gebbia N and Russo A: EGFR genomic alterations in cancer: Prognostic and predictive values. Front Biosci (Elite Ed) 3: 879-887, 2011.

7. Sharma MR and Schilsky RL: GI cancers in 2010: New standards and a predictive biomarker for adjuvant therapy. Nat Rev Clin Oncol 8: 70-72, 2011.

8. Abramson $\mathrm{V}$ and Arteaga CL: New strategies in HER2-overexpressing breast cancer: Many combinations of targeted drugs available. Clin Cancer Res 17: 952-958, 2011.

9. Seshacharyulu P, Ponnusamy MP, Haridas D, Jain M, Ganti AK and Batra SK: Targeting the EGFR signaling pathway in cancer therapy. Expert Opin Ther Targets 16: 15-31, 2012.

10. Pandya K, Meeke K, Clementz AG, Rogowski A, Roberts J, Miele L, Albain KS and Osipo C: Targeting both Notch and ErbB-2 signalling pathways is required for prevention of ErbB-2-positive breast tumour recurrence. Br J Cancer 105: 796-806, 2011.

11. Konecny GE, Pegram MD, Venkatesan N, Finn R, Yang G, Rahmeh M, Untch M, Rusnak DW, Spehar G, Mullin RJ, et al: Activity of the dual kinase inhibitor lapatinib (GW572016) against HER-2-overexpressing and trastuzumab-treated breast cancer cells. Cancer Res 66: 1630-1639, 2006.

12. Wainberg ZA, Anghel A, Desai AJ, Ayala R, Luo T, Safran B, Fejzo MS, Hecht JR, Slamon DJ and Finn RS: Lapatinib, a dual EGFR and HER2 kinase inhibitor, selectively inhibits HER2-amplified human gastric cancer cells and is synergistic with trastuzumab in vitro and in vivo. Clin Cancer Res 16: $1509-1519,2010$

13. Satoh T, Xu RH, Chung HC, Sun GP, Doi T, Xu JM, Tsuji A, Omuro Y, Li J, Wang JW, et al: Lapatinib plus paclitaxel versus paclitaxel alone in the second-line treatment of HER2-amplified advanced gastric cancer in Asian populations: TyTAN-a randomized, phase III study. J Clin Oncol 30: 2039-2049, 2014.

14. Shimoyama S: Unraveling trastuzumab and lapatinib inefficiency in gastric cancer: Future steps (Review). Mol Clin Oncol 2: 175-181, 2014.

15. Cenciarelli C, Marei HE, Zonfrillo M, Pierimarchi P, Paldino E, Casalbore P, Felsani A, Vescovi AL, Maira G and Mangiola A: PDGF receptor alpha inhibition induces apoptosis in glioblastoma cancer stem cells refractory to anti-Notch and anti-EGFR treatment. Mol Cancer 13: 247, 2014.

16. Chen T, Yang K, Yu J, Meng W, Yuan D, Bi F, Liu F, Liu J, Dai B, Chen $\mathrm{X}$, et al: Identification and expansion of cancer stem cells in tumor tissues and peripheral blood derived from gastric adenocarcinoma patients. Cell Res 22: 248-258, 2012.

17. Jiang J, Zhang Y, Chuai S, Wang Z, Zheng D, Xu F, Zhang Y, Li C, Liang Y and Chen Z: Trastuzumab (herceptin) targets gastric cancer stem cells characterized by CD90 phenotype. Oncogene 31: 671-682, 2012.

18. Xu G, Shen J, Ou Yang X, Sasahara M and Su X: Cancer stem cells: The 'heartbeat' of gastric cancer. J Gastroenterol 48: 781-797, 2013.

19. Kim TH and Shivdasani RA: Notch signaling in stomach epithelial stem cell homeostasis. J Exp Med 208: 677-688, 2011.

20. Takebe N, Nguyen D and Yang SX: Targeting notch signaling pathway in cancer: Clinical development advances and challenges. Pharmacol Ther 141: 140-149, 2014.
21. Cenciarelli C, Marei HE, Zonfrillo M, Casalbore P, Felsani A, GiannettiS,Trevisi G,Althani A and Mangiola A: The interference of Notch1 target Hes1 affects cell growth, differentiation and invasiveness of glioblastoma stem cells through modulation of multiple oncogenic targets. Oncotarge 8: 17873-17886, 2017.

22. Liu ZH, Dai XM and Du B: Hes1: A key role in stemness, metastasis and multidrug resistance. Cancer Biol Ther 16: 353-359, 2015.

23. Livak KJ and Schmittgen TD: Analysis of relative gene expression data using real-time quantitative PCR and the 2(-Delta Delta C(T)) method. Methods 25: 402-408, 2001.

24. Yin B, Zeng Y, Liu G, Wang X, Wang P and Song Y: MAGE-A3 is highly expressed in a cancer stem cell-like side population of bladder cancer cells. Int J Clin Exp Pathol 7: 2934-2941, 2014.

25. Liu J, Ma L, Xu J, Liu C, Zhang J, Liu J, Chen R and Zhou Y: Spheroid body-forming cells in the human gastric cancer cell line MKN-45 possess cancer stem cell properties. Int J Oncol 42: 453-459, 2013.

26. Lee J, Kotliarova S, Kotliarov Y, Li A, Su Q, Donin NM, Pastorino S, Purow BW, Christopher N, Zhang W, et al: Tumor stem cells derived from glioblastomas cultured in bFGF and EGF more closely mirror the phenotype and genotype of primary tumors than do serum-cultured cell lines. Cancer Cell 9: 391-403, 2006.

27. Bao S, Wu Q, McLendon RE, Hao Y, Shi Q, Hjelmeland AB, Dewhirst MW, Bigner DD and Rich JN: Glioma stem cells promote radio-resistance by preferential activation of the DNA damage response. Nature 444: 756-760, 2006.

28. Li LC, Wang DL, Wu YZ, Nian WQ, Wu ZJ, Li Y, Ma HW and Shao JH: Gastric tumor-initiating CD44+ cells and epithelial-mesenchymal transition are inhibited by $\gamma$-secretase inhibitor DAPT. Oncol Lett 10: 3293-3299, 2015.

29. Yamaguchi H, Chang SS, Hsu JL and Hung MC: Signaling cross-talk in the resistance to HER family receptor targeted therapy. Oncogene 33: 1073-1081, 2014.

30. Wang P, Shu B, Xu Y, Zhu J, Liu J, Zhou Z, Chen L, Zhao J, Liu X, Qi S, et al: Basic fibroblast growth factor reduces scar by inhibiting the differentiation of epidermal stem cells to myofibroblasts via the Notch1/Jagged1 pathway. Stem Cell Res Ther 8: 114, 2017.

31. Aster JC and Blacklow SC: Targeting the Notch pathway: Twists and turns on the road to rational therapeutics. J Clin Oncol 30: 2418-2420, 2012.

32. Takebe N, Nguyen D and Yang SX: Targeting notch signaling pathway in cancer: Clinical development advances and challenges. Pharmacol Ther 141: 140-149, 2014.

33. Liu ZH, Dai XM and Du B: Hes1: A key role in stemness, metastasis and multidrug resistance. Cancer Biol Ther 16: 353-359, 2015

34. Hynes NE and MacDonald G: ErbB receptors and signaling pathways in cancer. Curr Opin Cell Biol 21: 177-184, 2009.

35. Burgess AW: EGFR family: Structure physiology signalling and therapeutic targets. Growth Factors 26: 263-274, 2008.

36. Yarden Y and Sliwkowski MX: Untangling the ErbB signalling network. Nat Rev Mol Cell Biol 2: 127-137, 2001.

37. Arasada RR, Amann JM, Rahman MA, Huppert SS and Carbone DP: EGFR blockade enriches for lung cancer stem-like cells through Notch3-dependent signaling. Cancer Res 74: 5572-5584, 2014

38. Xie M, Zhang L, He CS, Xu F, Liu JL, Hu ZH, Zhao LP and Tian Y: Activation of Notch-1 enhances epithelial-mesenchymal transition in gefitinib-acquired resistant lung cancer cells. J Cell Biochem 113: 1501-1513, 2012.

39. Morishita A, Gong J and Masaki T: Targeting receptor tyrosine kinases in gastric cancer. World J Gastroenterol 20: 4536-4545, 2014.

40. Wang B, Lindley LE, Fernandez-Vega V, Rieger ME, Sims AH and Briegel KJ: The T box transcription factor TBX2 promotes epithelial-mesenchymal transition and invasion of normal and malignant breast epithelial cells. PLoS One 7: e41355, 2012.

41. Houthuijzen JM, Daenen LG, Roodhart JM and Voest EE: The role of mesenchymal stem cells in anti-cancer drug resistance and tumour progression. Br J Cancer 106: 1901-1906, 2012.

42. Chockalingam S and Ghosh SS: Amelioration of cancer stem cells in macrophage colony stimulating factor-expressing U87MG-human glioblastoma upon 5-fluorouracil therapy. PLoS One 8: e83877, 2013 